\title{
Cynandione A inhibits lipopolysaccharide-induced cell adhesion via suppression of the protein expression of VCAM-1 in human endothelial cells
}

\author{
KEUN HYUNG PARK ${ }^{1}$, JIYOUNG KIM ${ }^{1}$, EUNJOO H. LEE ${ }^{2}$ and TAE HOON LEE ${ }^{1,2}$ \\ ${ }^{1}$ Graduate School of Biotechnology and College of Life Science; ${ }^{2}$ Graduate School of East-West Medical Sciences, \\ Kyung Hee University, Yongin, Gyeonggi 446-701, Republic of Korea
}

Received June 12, 2017; Accepted January 8, 2018

DOI: $10.3892 /$ ijmm.2018.3376

\begin{abstract}
Cynandione A (CA) is one of the most active compounds in the roots of Cynanchum wilfordii, the extracts of which have been used extensively in East Asia to treat various diseases including anti-ischemic stroke. In the present study, the anti-adherent activity of CA in lipopolysaccharide (LPS)-stimulated human umbilical vascular endothelial cells (HUVECs) was investigated. CA markedly reduced the expression of vascular adhesion molecule-1 (VCAM-1) by LPS in HUVECs. The results also demonstrated that CA significantly reduced the expression of pro-inflammatory and chemoattractant cytokines, including interleukin (IL)-1 $\beta$, IL-6, IL-8, monocyte chemoattractant protein-1 and tumor necrosis factor- $\alpha$, in LPS-activated human endothelial cells. CA inhibited the phosphorylation of mitogen-activated protein kinases, including the extracellular signal-regulated kinase $1 / 2$ and p38 kinases. It was found that CA decreased the IKK/IкB- $\alpha$ phosphorylation of inhibitor of nuclear factor $(N F)-\kappa B$ kinase/inhibitor of NF- $\mathrm{B}-\alpha$, suppressed translocation of the $\mathrm{NF}-\kappa \mathrm{B}$ p65 subunit into the nucleus and inhibited the transcriptional activity of $\mathrm{NF}-\kappa \mathrm{B}$. CA also decreased human monocyte cell adhesion to endothelial cells in LPS-stimulated conditions. These results demonstrated that CA inhibited the protein expression of VCAM-1 and pro-inflammatory cytokines by suppressing the transcriptional activity of $\mathrm{NF}-\kappa \mathrm{B}$. The results also suggested that CA may be important in the development of anti-inflammatory drugs by inhibiting the expression of cell adhesion molecules.
\end{abstract}

Correspondence to: Professor Eunjoo H. Lee or Professor Tae Hoon Lee, Graduate School of East-West Medical Sciences, Kyung Hee University, 1 Seocheon-dong, Yongin, Gyeonggi 446-701, Republic of Korea

E-mail: ehwang@khu.ac.kr

E-mail: thlee@khu.ac.kr

Present address: Dr Keun Hyung Park, Skin Research Institute, IEC Korea, Suwon, 443-813, Republic of Korea

Key words: inflammation, adhesion, nuclear factor- $\kappa \mathrm{B}$, mitogen-activated protein kinase pathway, cynandione A

\section{Introduction}

During vascular inflammation, numerous cytokines and adhesion molecules are overexpressed, which leads to the homing of various immune cells into tissues (1-3). Major events in the homing of immune cells include the migration of leukocytes from the blood, their firm adhesion to the vascular endothelium and transmigration across the vascular endothelium (4). Several types of adhesion molecule are important in the endothelium cell adhesion process. Among these cell adhesion molecules, vascular adhesion molecule-1 (VCAM-1/CD106) is known to be increased in human endothelial cells by pro-inflammatory cytokines, including tumor necrosis factor (TNF)- $\alpha$, interleukin (IL)-1 $\beta$, IL-6 and various inflammatory stimuli, including reactive oxygen species (ROS) and lipopolysaccharide (LPS) $(5,6)$. In addition, the phosphorylation of mitogen-activated protein kinases (MAPKs), phosphoinositide-3 kinase and the nuclear factor $(\mathrm{NF})-\kappa \mathrm{B}$ pathways regulates the activation of the gene expression of VCAM-1 $(3,7,8)$. According to previous reports, the $\mathrm{NF}-\kappa \mathrm{B}$ signaling pathway in human endothelial cells is important in regulating cell-to-cell adhesion in the inflammatory response by inflammatory mediators associated with increased expression of VCAM-1. NF- $\kappa \mathrm{B}$ protein is known to be a pivotal transcription factor for the expression of inflammatory cytokine-mediated VCAM-1 $(9,10)$. The progressive accumulation of leukocyte adhesion to the endothelium may cause vascular inflammation.

LPS is a lipoglycan and a known endotoxin present in the outer membrane of Gram-negative bacteria, which affects inflammation and immune cell activation in animals (11). The binding of LPS to toll-like receptor 4, present in immune cells, affects various signal transduction pathways, particularly the activation of inflammatory signaling pathways involving MAPK/inhibitor of NF- $\mathrm{NB}(\mathrm{I} \kappa \mathrm{B})$ kinase $(\mathrm{IKK}) / \mathrm{I} \kappa \mathrm{B} / \mathrm{NF}-\kappa \mathrm{B}(12)$. In non-stimulated conditions, the

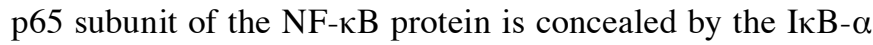
protein complex in the cytoplasm. However, when activated by external stimuli, including inflammatory molecules, the IKK- $\alpha / \beta$ subunit undergoes a successive phosphorylation cascade (13). Subsequently, the activated and released p65 subunit of $N F-\kappa B$ is translocated into the nucleus and binds to DNA promoter regions, increasing the transcription of 
inflammatory-mediated genes, including cell adhesion molecules $(14,15)$.

In ancient oriental medicine, active ingredients isolated from natural products and herbal extracts have been used to treat various diseases. Previous studies have shown that Cynanchum wilfordii root extracts have been used in traditional medicine for the therapeutic treatment and prevention of diseases, including vascular disease, arteriosclerosis and cancer (16-18). The active ingredient of $C$. wilfordii roots, cynandione A (CA; Fig. 1A), has been isolated in a number of studies $(19,20)$. In addition, several studies investigating the biological and pharmaceutical activity of CA have revealed it exhibits protective activity against toxicity by various stimulant agents in rat hepatocytes and cortical neurons (21-23). Among investigations on the inhibitory effects of CA on anti-inflammatory activity, few have reported on the molecular mechanism underlying the anti-adhesion effects of CA in vascular inflammation. In the present study, it was demonstrated that CA inhibited the mRNA and protein expression of VCAM-1 in LPS-induced human umbilical vascular endothelial cells (HUVECs). The results confirmed that CA inhibited the expression of pro-inflammatory cytokines, including IL-1 $\beta$, IL-6, IL-8, monocyte chemoattractant protein-1 (MCP-1) and TNF- $\alpha$, in LPS-activated HUVECs. It was also shown that CA inhibited the phosphorylation of IKK and the transcriptional activity of $\mathrm{NF}-\kappa \mathrm{B}$ via downregulating the phosphorylation of MAPKs. Finally, the anti-adhesion activity of CA was confirmed using FITC-labeled immune cells. These findings may be beneficial for the development of a useful therapeutic agent for vascular inflammatory diseases, including atherosclerosis.

\section{Materials and methods}

Materials and cell culture. Human endothelial cells were cultured in medium 199 containing fetal bovine serum (FBS) and antibiotics (penicillin/streptomycin) prepared by Gibco; Thermo Fisher Scientific, Inc. (Waltham, MA, USA). Anti-human VCAM-1 (1:200; cat no. SC-13160) and anti-b-actin (1:200; cat no. SC-47778) antibodies were purchased from Santa Cruz Biotechnology, Inc. (Santa Cruz, CA, USA). The antibodies targeting phosphorylated (p-)c-Jun N-terminal kinase (JNK; 46 kDa, 1:1,000; cat no. MAB1205), p-extracellular signal-regulated kinase (ERK)1/2 (44,42 kDa, 1:1,000; cat no. MAB18251), p-p38 (38 kDa, 1:500; cat no. MAB8691), JNK, ERK and p38 antibodies were from R\&D Systems, Inc. (Minneapolis, MN, USA). Antibodies against p-IKK $\alpha / \beta(87,85 \mathrm{kDa}, 1: 1,000$; cat no. \#2697) p-IкB- $\alpha$ (40 kDa, 1:2,000; cat no. \#9246), anti-p65 (65 kDa, 1:1,000; cat no. \#8242) and all non-phosphorylated antibodies were prepared and acquired from Cell Signaling Technology, Inc. (Beverly, MA, USA). Signal inhibitors AG490 (a Janus kinase 2 inhibitor), U0126 (an MAPK kinase/ERK inhibitor) and SB203580 (a p38 inhibitor) were purchased from Cell Signaling Technology, Inc., and PDTC (an NF- $\kappa \mathrm{B}$ inhibitor) was from EMD Millipore (Billerica, MA, USA). The primers used for polymerase chain reaction (PCR) analysis were prepared from Bioneer Corporation (Daejeon, Korea). Other chemicals were purchased commercially from Sigma-Aldrich; Merck Millipore (Darmstadt, Germany).
The HUVECs (BMS, Seoul, Korea) were grown over three to six passages in cell culture medium at $37^{\circ} \mathrm{C}$ under a humidified 95 and $5 \%(\mathrm{v} / \mathrm{v})$ mixture of air and $\mathrm{CO}_{2}$ supplemented with $10 \% \mathrm{FBS}, 100 \mathrm{U} / \mathrm{ml}$ penicillin, $100 \mu \mathrm{g} / \mathrm{ml}$ streptomycin, $3 \mathrm{ng} / \mathrm{ml}$ bFGF and $5 \mathrm{U} / \mathrm{ml}$ heparin. The U937 human monocyte-like cell line (American Type Culture Collection, Manassas, VA, USA; CRL-1593.2 $2^{\mathrm{TM}}$ ) was also cultured in complete RPMI1640 cell culture medium under the same conditions as the HUVECs.

Cell viability assay. The HUVECs $\left(1 \times 10^{4}\right.$ cells/well) were removed from the cell growth medium and incubated in serum-free medium for $18 \mathrm{~h}$. These cells were then incubated with or without LPS $(1 \mu \mathrm{g} / \mathrm{ml})$ in the presence of each indicated CA concentration $(0,5,10,20$ and $40 \mu \mathrm{M})$ for $48 \mathrm{~h}$ at $37^{\circ} \mathrm{C}$ Cell viability was measured by the addition of $50 \mu \mathrm{g} / \mathrm{ml} \mathrm{3-(4,5-dimetnythiazol-2-yl)-2,5-diphenyl-thetazo-}$ lium bromide (MTT). Following incubation for $2 \mathrm{~h}$, purple formazan had formed, the supernatants were removed from each well and $100 \mu \mathrm{l}$ DMSO was added to completely dissolve the formazan crystals. Data was quantified by measuring the absorbance at $540 \mathrm{~nm}$ using a spectrophotometric multi-well microplate reader (Multiskan MS; Thermo Fisher Scientific, Inc.).

Reverse transcriptase-polymerase chain reaction (RT-PCR) analysis. Total RNA from HUVECs was isolated according to the manufacturer's protocol using an RNA-Bee isolation kit (Tel-Test, Inc., Friendswood, TX, USA). The cDNA was prepared by reverse transcription using $\mathrm{M}-\mathrm{MuLV}$ reverse transcriptase (Fermentas; Thermo Fisher Scientific, Inc.) and PCR amplification was performed according to the manufacturer's protocol using an AccuPower PCR PreMix (Bioneer Coporation; Daejeon, Korea). Using $250 \mathrm{ng}$ of cDNA and $10 \mathrm{pmole} / \mu \mathrm{l}$ of forward and reverse primers, PCR was performed as follows: Intercellular adhesion molecule (ICAM)-1 (556 bp, annealing temp. $60^{\circ} \mathrm{C}, 25$ cycles), forward 5'-CAGTGACCA TCTACAGCTTTCCGG-3' and reverse 5'-GCTGCTACCACA GTGATGATGACAA-3'; VCAM-1 (742 bp, annealing temp. $62^{\circ} \mathrm{C}, 32$ cycles), forward 5'-AATTTATGTGTGTGAAGG AG-3' and reverse 5'-GCATGTCATATTCACAGAA-3'; IL-1 $\beta$ (264 bp, annealing temp. $62^{\circ} \mathrm{C}, 33$ cycles), forward $5^{\prime}-\mathrm{GGA}$ TATGGAGCAACAACTGG-3' and reverse 5'-ATGTACCAG TTGGGGAACTG-3'; IL-6 (497 bp, annealing temp. $54^{\circ} \mathrm{C}$, 30 cycles), forward 5'-TGACAAACAAATTCGGTACAT CC-3' and reverse 5'-ATCTGAGGTGCCCATGCTAC-3'; IL-8 (292 bp, annealing temp. $63^{\circ} \mathrm{C}, 28$ cycles), forward $5^{\prime}$-ATGACT TCCAAGCTGGCCGTGGCT-3' and reverse 5'-TCTCAG CCCTCTTCAAAAACTTCTC-3'; MCP-1 (261 bp, annealing temp. $62^{\circ} \mathrm{C}, 30$ cycles), forward 5'-TCTGTGCCTGCTGCT CATAG-3' and reverse 5'-TTTGCTTGTCCAGGTGGTCC-3'; and TNF- $\alpha$ (444 bp, annealing temp. $62^{\circ} \mathrm{C}, 32$ cycles), forward 5'-GACTGACAAGCCTGTAGCCCATGTTGTTGTAGCA-3' and reverse 5'-GCAATGATCCCAAAGTAGACCTGCCCA GAC-3'; $\beta$-actin (482 bp, annealing temp. $58^{\circ} \mathrm{C}, 28$ cycles), forward 5'-TGAGACCTTCAACACCCCAG-3' and reverse 5'-CACTGTGTTGGCGTACAGGT-3'. Each band intensity was detected for all bands and standardization relative $\beta$-actin and measured using ImageJ software (Ver.1.51e; National Institutes of Health, Bethesda, MD, USA). 
Western blot analysis. The HUVECs were treated with cell lysis buffer to obtain cell extracts. Protein quantity was determined using a Bradford assay kit according to the manufacturer's protocol (Thermo Fisher Scientific, Inc.) and equal quantities of protein $(30 \mu \mathrm{g})$ were separated by performing electrophoresis on 6-15\% SDS-PAGE gels. The proteins were then transferred onto a nitrocellulose membrane, which was gently agitated and washed with $3-5 \%$ non-fat dry milk in Tris-buffered saline-Tween-20 buffer, containing $0.5 \mathrm{~mol} / \mathrm{l}$ Tris- $\mathrm{HCl}(\mathrm{pH} 7.5), 0.15 \mathrm{~mol} / \mathrm{l} \mathrm{NaCl}$ and $1 \mathrm{~g} / \mathrm{l}$ Tween-20. The membrane was incubated with each primary antibody overnight at $4^{\circ} \mathrm{C}$ according to manufacturer's protocol followed by the secondary antibodies $(1: 2,000) 2 \mathrm{~h}$ at room temperature which was then visualized through enhanced chemiluminescence and exposure to X-ray films. The intensity of each band was measured as aforementioned using ImageJ software.

Luciferase reporter assay. VCAM-1 promoter region information was obtained by searching human VCAM-1 gene sequences (www.ncbi.nlm.nih.gov/), following which the pVCAM-1/Luc plasmid construct, containing the $-1,350$ to +45 bp promoter region in the pGL3-basic vector (Promega, Madison, WI, USA), was constructed. The HUVECs were transiently transfected with empty vector (pGL3-basic), constructed $\mathrm{pVCAM}-1 / \mathrm{Luc}$ or $\mathrm{pNF}-\kappa \mathrm{B} /$ Luc (Stratagene; Agilent Technologies, Inc., Santa Clara, CA, USA) using Lipofectamine $^{\mathrm{TM}} 2000$ reagent (Stratagene; Agilent Technologies, Inc.). Following $24 \mathrm{~h}$ of transfection, cell extracts were prepared and luciferase activity was detected using the Renilla luciferase assay kit according to the manufacturer's protocol (Promega). Data was quantified by measuring the fluorescence intensity at $480 \mathrm{~nm}$ (excitation) and $560 \mathrm{~nm}$ (emission) using a fluorescence multi-well microplate reader (Wallac 1420 Victor 2; PerkinElmer, Inc., Waltham, MA, USA).

Fluorescence microscopy analysis. Growth medium was removed from the HUVECs and serum-free medium containing CA was stimulated with or without LPS $(1 \mu \mathrm{g} / \mathrm{ml})$ for $2 \mathrm{~h}$. The HUVECs were incubated overnight at $4^{\circ} \mathrm{C}$ with the FITC-labeled NF-kB p65 antibody [(F-6) Alexa Fluor ${ }^{\mathrm{R}} 488$; 65 kDa; 1:100; cat. no. SC-8008; Santa Cruz Biotechnology, Inc.], and then treated with $3.7 \%$ paraformaldehyde solution to preserve the cell state and then washed in PBS. The fluorescence intensities were analyzed for the distribution and translocation of $\mathrm{p} 65$ protein. The fixed cells were stained with mounting solution containing 4'-6-diamidino-2-phenylindole to indicate the location of nuclei in the cells.

Cell adhesion assay. The U937 cell line has the monocyte phenotype and can therefore be used in monocyte adhesion experiments, which were performed as described in a previous study (24). The HUVECs were cultured to confluence in 6-well plates and pre-treated with each signal inhibitor $(10 \mu \mathrm{M})$ with $\mathrm{CA}$ at the indicated concentrations for $12 \mathrm{~h}$. The U937 cells were cultured in $1 \% \mathrm{FBS}$ and $5 \mu \mathrm{g} / \mathrm{ml}$ calcein-AM (Invitrogen; Thermo Fisher Scientific, Inc.) RPMI medium at $37^{\circ} \mathrm{C}$ for $30 \mathrm{~min}$ for fluorescent labeling. The fluorescence-labeled U937 cells $\left(1 \times 10^{7}\right)$ were re-suspended in HUVEC culture medium and then incubated for $30 \mathrm{~min}$ with the pre-cultured
HUVECs. The non-adherent fluorescent U937 cells were washed with PBS and the fluorescence intensity of attached cells was quantitated using a Wallace Victor2 1420 Multi-label counter (PerkinElmer, Inc.).

Statistical analysis. Unless otherwise stated, all experiments were performed with triplicate samples and repeated at least three times. The data are presented as the mean \pm standard deviation and statistical comparisons between groups were performed using one-way analysis of variance followed by Student's t-tests using SigmaPlot software (version 11; Systat Software Inc., San Jose, CA, USA). P $<0.05$ was considered to indicate a statistically significant differences.

\section{Results}

CA inhibits the LPS-induced $m R N A$ and protein expression of VCAM-1 in HUVECs. The present study examined cell viability using an MTT assay to determine the in vitro experimental concentration of CA. As shown in Fig. 1B and C, the cells were treated with $\mathrm{CA}$ for $24 \mathrm{~h}$ in the presence or absence of LPS. It was confirmed that treatment of the HUVECs with CA was not toxic up to a concentration of $40 \mu \mathrm{M}$ CA. Based on this information, the mRNA expression levels of ICAM-1 and VCAM-1 were examined in the LPS-induced HUVECs at the indicated concentrations of CA. The results showed that CA did not affect the mRNA expression of ICAM-1, however, the mRNA expression of VCAM-1 was decreased in a concentration-dependent manner in the LPS-induced HUVECs (Fig. 1D). Subsequently, the results of western blot analysis showed that the protein expression levels of ICAM-1 and VCAM-1 were significantly increased by LPS stimulation in the HUVECs. Similar to the mRNA expression levels, CA markedly inhibited the protein expression of VCAM- 1 and had no effect on the protein expression level of ICAM-1 in the LPS-stimulated HUVECs (Fig. 1E).

CA inhibits the pro-inflammatory cytokine expression in LPS-stimulated HUVECs. The present study subsequently examined the activity of CA on the mRNA expression levels of IL-1 $\beta$, IL-6, IL-8, MCP-1 and TNF- $\alpha$ in HUVECs. In the LPS-stimulated HUVECs, the gene expression levels of the pro-inflammatory cytokines IL- $1 \beta$, IL-6, IL-8, MCP-1 and TNF- $\alpha$ increased $\sim 6$-9-fold, compared with those in the untreated control. In the CA-treated HUVECs, the mRNA level of each pro-inflammatory cytokine was significantly decreased in a CA concentration-dependent manner (Fig. 2).

Role of MAPKs in the inhibition of VCAM-1 by CA in LPS-induced HUVECs. To reveal the molecular mechanism for the inhibitory activity of CA on the expression of VCAM-1 and pro-inflammatory cytokines, the present study analyzed the phosphorylation of MAPKs in the LPS-induced HUVECs with or without CA exposure. The effects of CA and commercial signal inhibitors on the mRNA expression levels of VCAM-1 and pro-inflammatory cytokines were examined in the LPS-activated HUVECs. The HUVECs were treated with AG490 (a Janus kinase inhibitor), U0126 (an MEK/ERK inhibitor), SB203580 (a p38 inhibitor), PDTC (an NF-кB inhibitor) and at the indicated concentration of CA. After $1 \mathrm{~h}$, 
<smiles>CC(=O)c1ccc(O)c(-c2c(O)ccc(O)c2C(C)=O)c1O</smiles>
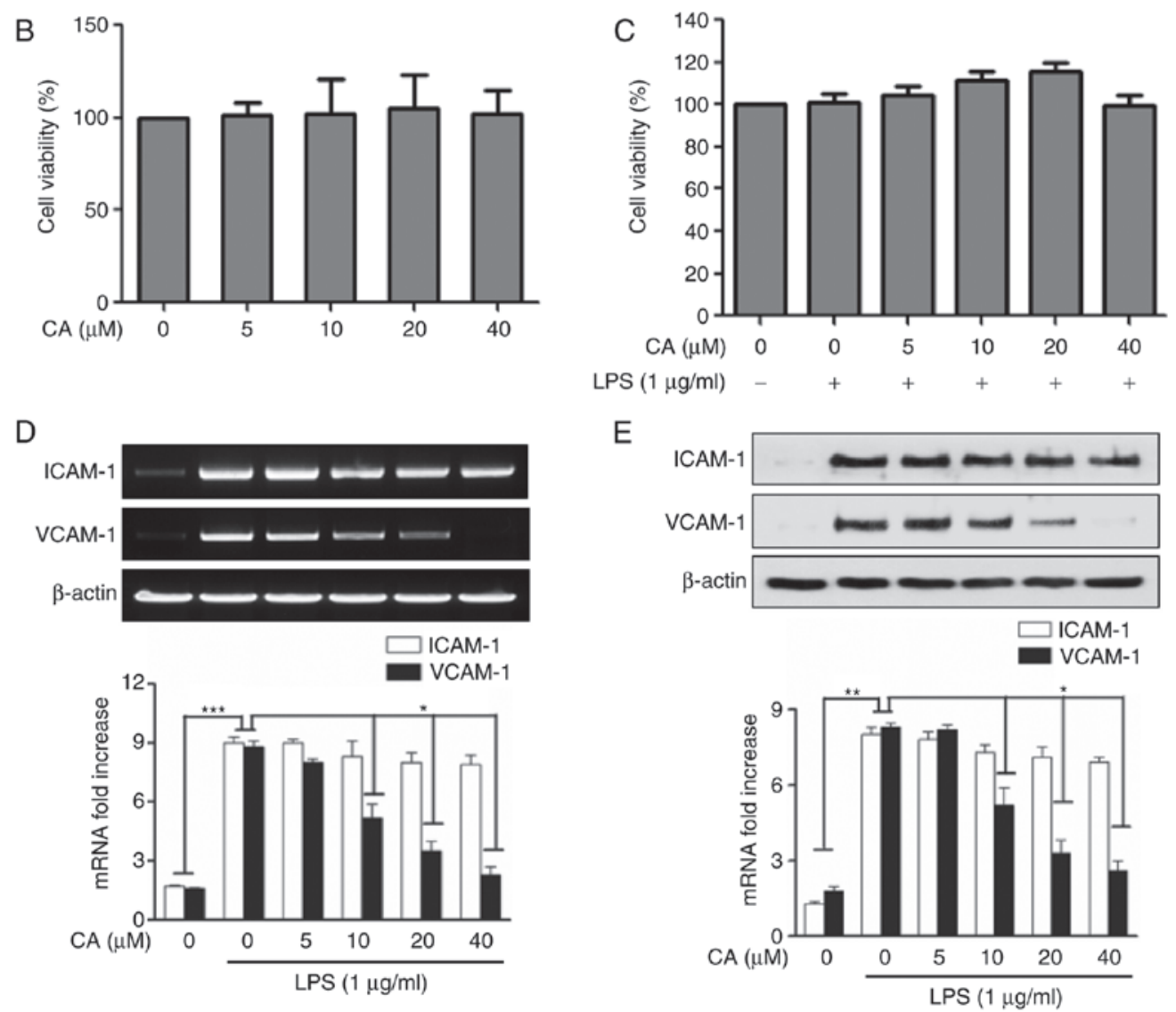

Figure 1. Effects of CA on mRNA and protein expression of cell adhesion molecules in LPS-stimulated human umbilical vascular endothelial cells. (A) Chemical structure of CA. Cell viability assessment of (B) CA-treated cells and (C) cells treated with CA+LPS was evaluated using a 3-(4,5-dimetnythiazol-2-yl)-2,5-diphenyl-thetazolium bromide assay. The cells were cultured for $24 \mathrm{~h}$ at each indicated CA concentration and the results were measured by optical density value. Results are shown as a percentage of the negative control, which was treated with medium alone. Each value is presented as the mean \pm standard deviation. (D) Reverse transcription-quantitative polymerase chain reaction experiments were performed to compare mRNA levels of cell adhesion molecules. The values for intensity are presented as the mean \pm standard deviation of three independent experiments. ${ }^{* * *} \mathrm{P}<0.001$, compared with treatment with medium alone; ${ }^{*} \mathrm{P}<0.05$, compared with treatment with LPS alone. (E) Western blot analyses were performed to compare the expression levels of cell adhesion molecules. The intensity of each band was compared and data are presented as a graph. The values for intensity are presented the mean \pm standard deviation from three independent experiments. ${ }^{* *} \mathrm{P}<0.01$, compared with treatment with medium alone; ${ }^{*} \mathrm{P}<0.05$, compared with treatment with LPS alone. CA, cynandione A; LPS, lipopolysaccharide; ICAM-1, intercellular adhesion molecule-1; VCAM-1, vascular adhesion molecule-1.

the incubated HUVECs in the commercial signal inhibitors and CA were stimulated with LPS. Our data showed that, with the exception of AG490, all the commercial signal inhibitors inhibited the mRNA and protein levels of VCAM-1 in LPS-induced HUVECs. CA inhibited the mRNA and protein expression levels of VCAM-1 to levels similar to those in the U0126, SB203580 and PDTC treatment groups (Fig. 3A). This result indicated that key signal inhibitors, including U0126, SB203580 and PDTC, inhibited the expression of VCAM-1 and the upstream signaling molecules of VCAM-1 in the LPS-stimulated HUVECs.
Subsequently, the present study examined the effect of CA on cellular signaling molecules activated by LPS-stimulation in HUVECs. The inhibitory activity of CA on the phosphorylation of the most well known MAPK pathways, including JNK, ERK1/2 and p38 proteins, were then confirmed in LPS-induced HUVECs. The results indicated that CA decreased the phosphorylation of ERK1/2 and p38 proteins in the LPS-stimulated HUVECs in a concentration-dependent manner, whereas CA had minimal effect on the phosphorylation of JNK (Fig. 3B). These results suggested that the inhibitory activity of CA on the expression of VCAM-1 may be associated with 

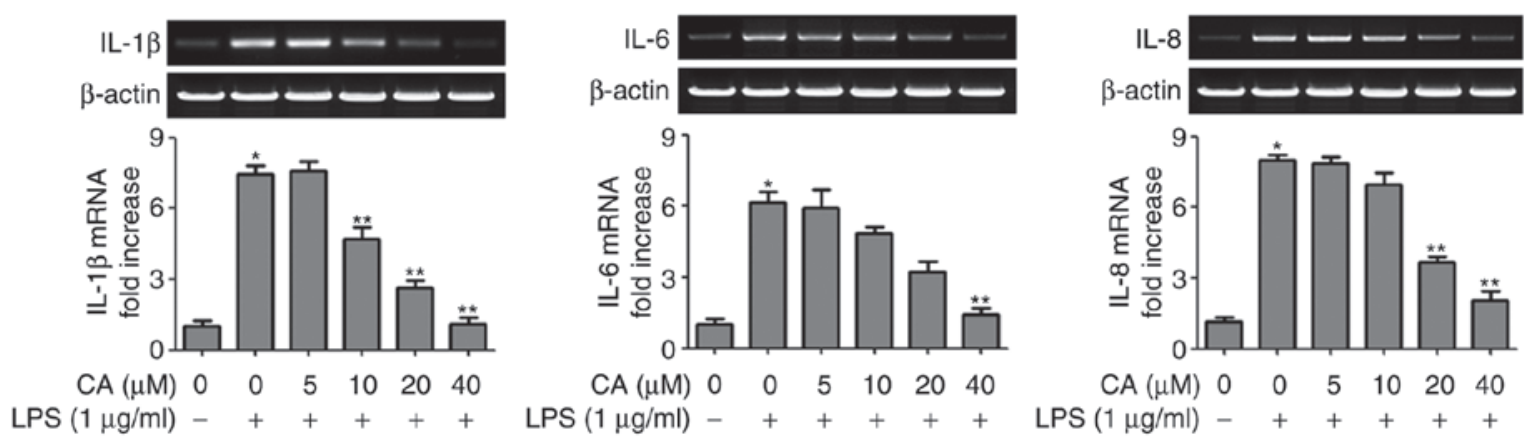

$\mathrm{CA}(\mu \mathrm{M}) 0 \begin{array}{llllll}0 & 0 & 5 & 10 & 20 & 40\end{array}$ LPS $(1 \mu \mathrm{g} / \mathrm{ml})-+++++$

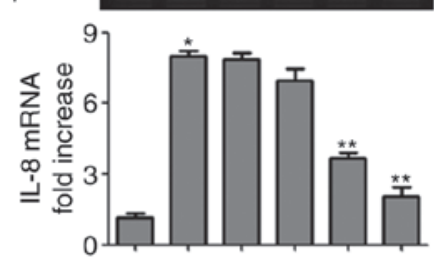

$\mathrm{CA}(\mu \mathrm{M}) \quad 0 \quad 0 \quad 5 \quad 10 \quad 20 \quad 40$ LPS $(1 \mu \mathrm{g} / \mathrm{ml})-+++++$

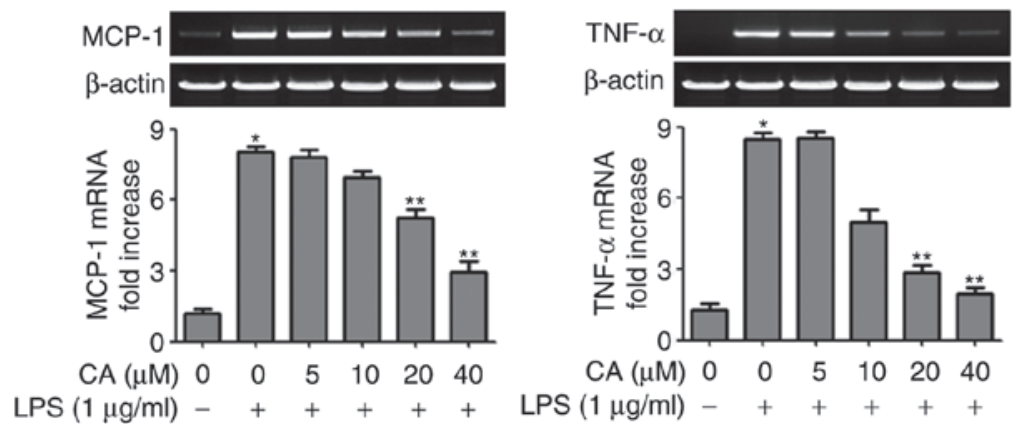

Figure 2. Inhibitory effect of CA on expression of LPS-induced pro-inflammatory cytokines and chemoattractant cytokines in HUVECs. Reverse transcription polymerase chain reaction analyses were performed to compare the mRNA expression levels of IL-1 $\beta$, IL-6, IL-8, MCP-1 and TNF- $\alpha$. HUVECs were pretreated with CA at the indicated concentration for $1 \mathrm{~h}$ and then treated with LPS. The values for intensity are presented as the mean \pm standard deviation of three independent experiments. ${ }^{*} \mathrm{P}<0.01$, compared with treatment with medium alone; ${ }^{* *} \mathrm{P}<0.05$, compared with treatment with LPS alone. HUVECs, human umbilical vascular endothelial cells; CA, cynandione A; LPS, lipopolysaccharide; IL, interleukin; MCP-1, monocyte chemoattractant protein-1; TNF- $\alpha$, tumor necrosis factor- $\alpha$.

inhibition of the phosphorylation of ERK1/2 and p38 kinase in LPS-stimulated HUVECs.

To analyze the effect of CA on MAPK downstream signaling pathways, the present study confirmed the activity of CA on the phosphorylation of IKK/I $\mathrm{B}-\alpha$ signal transduction. The NF- $\kappa \mathrm{B}$ p 65 subunit is an important transcription factor for the expression of VCAM-1 in LPS-stimulated HUVECs (25). The nuclear translocation of the p65 subunit is activated by the phosphorylation of I $\mathrm{B}-\alpha$. Therefore, the effect of CA on the phosphorylation of IKK was examined. The results demonstrated that CA significantly inhibited the phosphorylation of IKK in LPS-stimulated HUVECs. In addition, it was found that $\mathrm{CA}$ inhibited the phosphorylation of $\mathrm{I} \kappa \mathrm{B}-\alpha$ protein and suppressed the degradation of $\mathrm{I} \kappa \mathrm{B}-\alpha$ protein in the cell cytosolic fraction (Fig. 3C). These results suggested that CA significantly inhibited IKK/I $\kappa \mathrm{B}-\alpha$ signal transduction. The protein levels of p65 in the nucleus of cells treated with CA in the LPS-stimulated HUVECs were examined. In the nuclei of resting state cells, a basic level of p65 protein was identified, and in the cells treated with LPS alone, the nuclear translocation of p65 subunit was significantly increased. In cells stimulated with LPS in the presence of CA, it was confirmed that the protein expression of p65 was decreased in a concentration-dependent manner. The reduced protein expression of p65 was similar to that in the non-stimulated cells (Fig. 3D). The inhibitory activity of CA on the expression of VCAM-1 and pro-inflammatory cytokines may be due to the decrease in nuclear p65 translocation through inhibition of the phosphorylation of MAPK/IKK/IкB- $\alpha$ and degradation of I $\mathrm{B} \mathrm{B}-\alpha$.
CA inhibits LPS-induced transcription of the VCAM-1 promoter and activation of $N F-\kappa B$. The present study investigated whether $\mathrm{CA}$ regulates the transcriptional activity of the VCAM-1 gene using VCAM-1 luciferase reporter gene constructs. The transfected HUVECs were treated with or without CA and then activated with LPS. Stimulation of the transfected cells with LPS promoted luciferase activity by 5-6-fold over that in the mock transfected cells. CA significantly reduced the LPS-activated induction of VCAM-1 in the transfected HUVECs at the transcriptional level (Fig. 4A). The regulatory activity of $\mathrm{CA}$ on transcriptional activation was examined using the established $\mathrm{pNF}-\kappa \mathrm{B} / \mathrm{Luc}$ reporter gene construct by LPS stimulation. As shown Fig. 4B, the results demonstrated that $\mathrm{CA}$ reduced transcriptional activity by LPS in a dose-dependent manner in the HUVECs transfected with the $\mathrm{pNF}-\kappa \mathrm{B} /$ Luc reporter gene construct. To analyze the activity of CA on the translocation of the p65 subunit into the nucleus, fluorescence microscopy was used to analyze the HUVECs treated with or without CA. It was confirmed that the fluorescence intensity in the nucleus was increased in cells treated with LPS alone, and a large quantity of p65-FITC antibody was present in the nucleus. By contrast, CA decreased fluorescence intensity in the nucleus by LPS stimulation, and a similar result was observed in the cells treated with PDTC, which is an NF- $\mathrm{B}$ specific signaling inhibitor (Fig. 4C).

CA inhibits the LPS-induced adhesion of U937 cells to HUVECs. The overproduction of cell adhesion molecules, including VCAM-1, by LPS likely increases the adherence of monocytes to endothelial cells in the early stages of vascular 

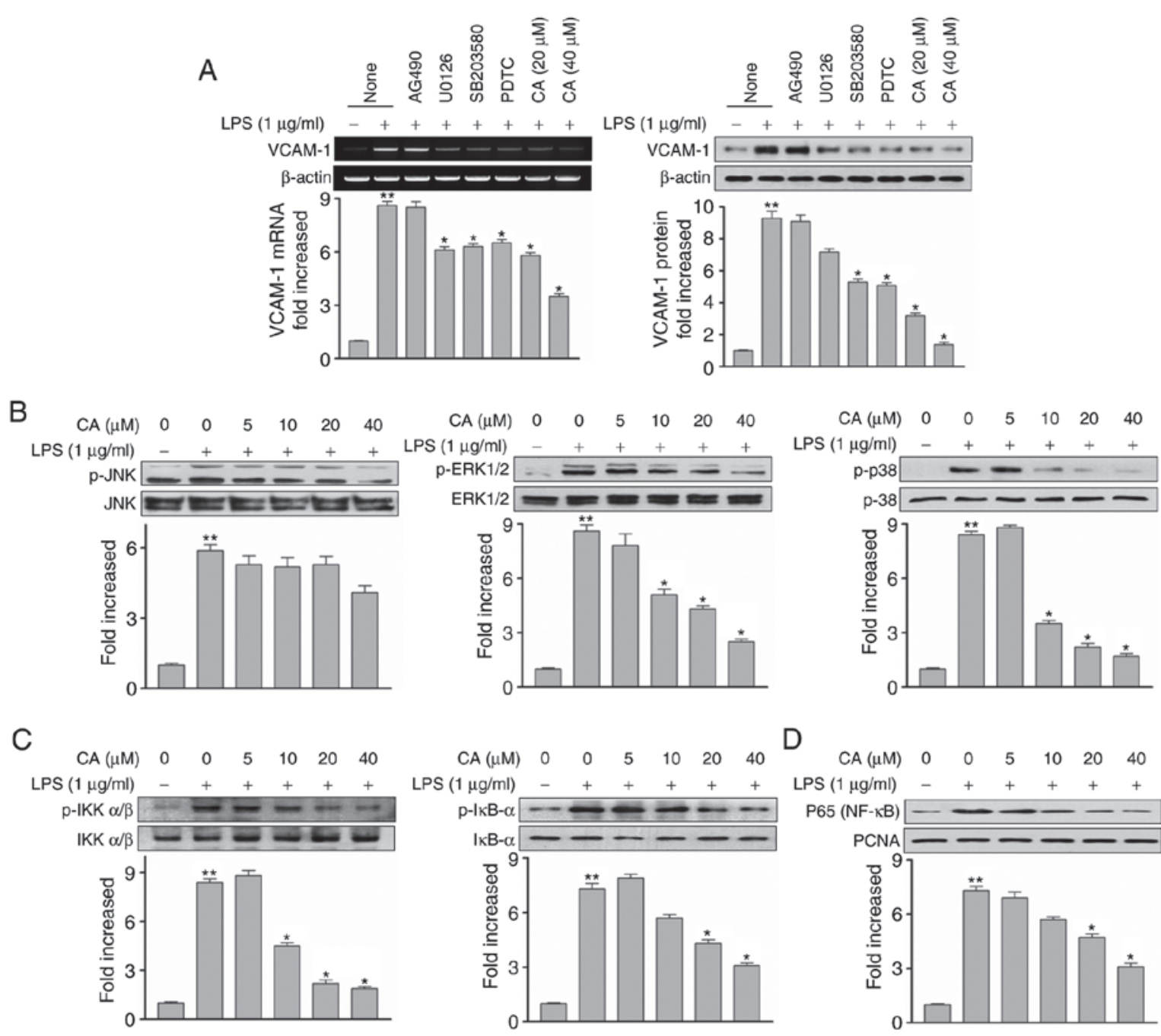

Figure 3. Effects of CA on the phosphorylation of mitogen-activated protein kinase/IKK/IкB and analysis of the translocation of p65 into the nucleus of LPS-activated HUVECs. (A) HUVECs were pretreated with the indicated signal inhibitor, AG490 $(10 \mu \mathrm{M})$, U0126 (10 $\mu \mathrm{M})$, SB203580 (10 $\mu \mathrm{M})$ or PDTC $(10 \mu \mathrm{M})$ for $1 \mathrm{~h}$, and stimulated with LPS with or without CA for $24 \mathrm{~h}$. mRNA and protein expression levels of VCAM-1 were detected using reverse transcription-quantitative polymerase chain reaction and western blot analyses, respectively. (B) HUVECs were starved for $6 \mathrm{~h}$ and then pre-treated with CA for $1 \mathrm{~h}$, followed by activation by LPS for $30 \mathrm{~min}$. Phosphorylation patterns were analyzed using western blot analysis. (C) HUVECs were starved for $6 \mathrm{~h}$ and then pre-treated with CA for $1 \mathrm{~h}$, followed by activation by LPS for $30 \mathrm{~min}$. The activation levels of p-IKK, p-IкB- $\alpha$, IKK and IкB- $\alpha$ were detected using western blot analysis. (D) HUVECs were starved for $6 \mathrm{~h}$ and then pre-treated with CA for $1 \mathrm{~h}$, followed by activation with LPS for $2 \mathrm{~h}$. Proteins in the nuclear extract fraction were prepared and NF- $\mathrm{kB}$ p65 subunit translocation levels were analyzed using western blot analysis. ${ }^{* *} \mathrm{P}<0.01$, compared with the negative control; "P<0.05, compared with treatment with LPS alone. HUVECs, human umbilical vascular endothelial cells; CA, cynandione A; LPS, lipopolysac-

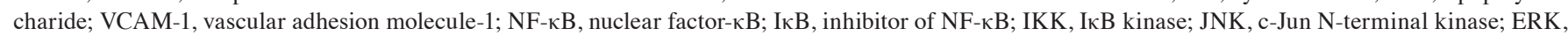
extracellular signal-regulated kinase; p-, phosphorylated.

inflammation. Therefore, in order to demonstrate the effect of CA on U937 cell adhesion to endothelial cells, the present study compared cell adhesion activity with various signal inhibitors in the presence of CA in the LPS-induced HUVECs. In the case of cells treated with LPS alone, the adhesion activity of the U937 cells was markedly increased compared with that in the control cells. The data showed that U0126, SB203580 and PDTC significantly inhibited U937 adhesion in the LPS-induced HUVECs, whereas AG490 had no effect. The LPS-activated HUVECs were treated with CA $(20$ or $40 \mu \mathrm{M})$, and it was shown that CA inhibited U937 adhesion to the HUVECs (Fig. 5). The data showed that CA inhibited the LPS-induced expression of VCAM-1 and suppressed U937 adhesion to HUVECs.

\section{Discussion}

In the present study, it was found that CA had anti-adhesive activity with regard to the adhesion of U937 monocytes to HUVECs via suppressing the expression of VCAM-1 and pro-inflammatory mediators in LPS-stimulated HUVECs. The inhibitory activity of CA on the expression of cell adhesion molecules was examined in LPS-induced HUVECs, and it was demonstrated that CA treatment inhibited the mRNA and protein expression levels of VCAM-1. The inhibition of VCAM-1 protein expression in LPS-induced HUVECs was due to the fact that $\mathrm{CA}$ inhibited the phosphorylation and degradation of $I \kappa \mathrm{B}-\alpha$, the activation of NF- $\kappa \mathrm{B}$, and the translocation of NF- $\kappa \mathrm{B}$ p 65 subunit to the nucleus. Therefore, the 

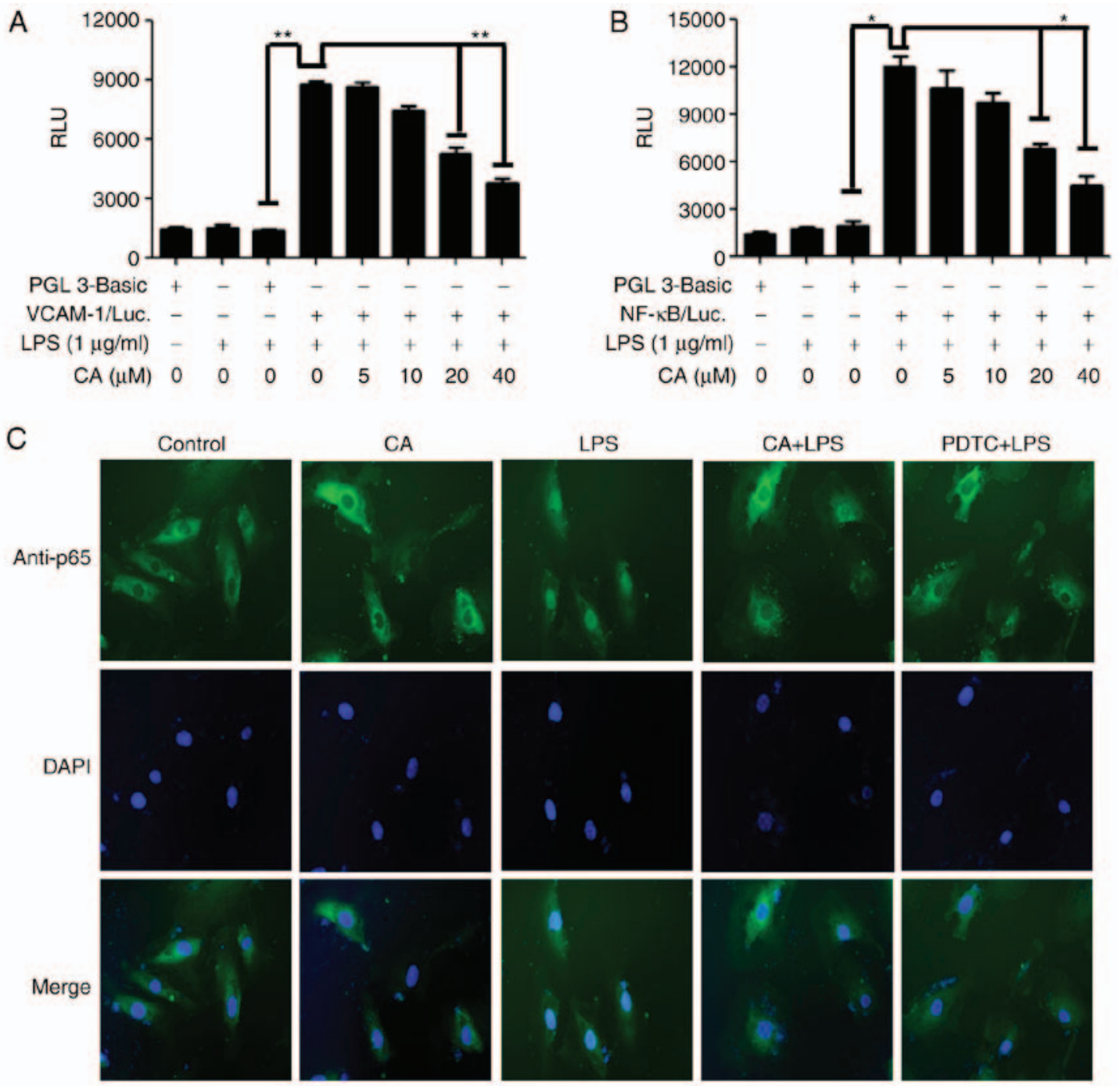

Figure 4. Effects of CA on the transcriptional activity of NF- $\mathrm{kB}$ and luciferase reporter genes in transiently transfected HUVECs. (A) pVCAM-1/Luc transfected HUVECs were cultured for $24 \mathrm{~h}$, and treated with indicated concentrations of CA and $1 \mu \mathrm{g} / \mathrm{ml} \mathrm{LPS}$ for $24 \mathrm{~h}$. The values for relative luciferase intensity are shown as the mean \pm standard deviation of three independent experiments $(\mathrm{n}=3) .{ }^{* * *} \mathrm{P}<0.01$, compared with the mock transfectant; ${ }^{* *} \mathrm{P}<0.01$, compared with treatment with LPS alone. (B) pNF-kB/Luc transfected HUVECs were cultured for $24 \mathrm{~h}$, and treated with indicated concentrations of CA and $1 \mu \mathrm{g} / \mathrm{ml} \mathrm{LPS}$ for $24 \mathrm{~h}$. The values for relative luciferase intensity are shown as the mean \pm standard deviation from three independent experiments $(\mathrm{n}=3)$. ${ }^{*} \mathrm{P}<0.05$, compared with the mock transfectant; ${ }^{*} \mathrm{P}<0.05$, compared with treatment with LPS alone. (C) Nuclear translocation of NF-кB p65 subunit was analyzed following CA $(40 \mu \mathrm{M})$ and PDTC $(10 \mu \mathrm{M})$ treatment for $1 \mathrm{~h}$. Cells were activated for $2 \mathrm{~h}$ with LPS and then observed via fluorescence microscopy (magnification, $\mathrm{x} 100$ ).

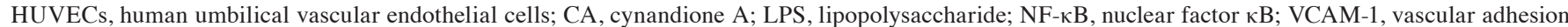
molecule-1; Luc, luciferase.
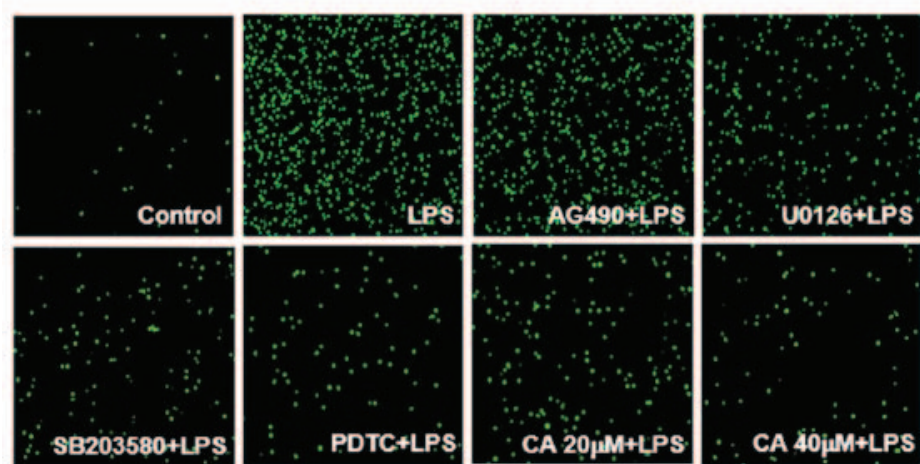

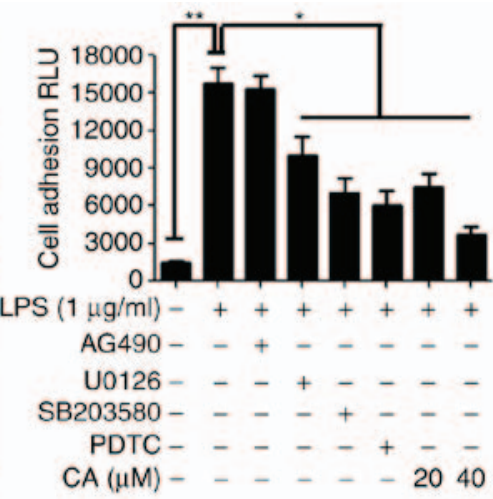

Figure 5. CA reduces U937 monocyte adhesion to endothelial cells. Cells were pretreated with or without each signal inhibitor (10 $\mu \mathrm{M})$ and CA at the indicated concentration for $12 \mathrm{~h}$. Adhesion of U937 cells to HUVECs was quantitatively measured by the fluorescence intensity of FITC-labeled U937 cells adherent to LPS-activated HUVECs and then observed via fluorescence microscopy (x40). The values for relative luciferase intensity are shown as the mean \pm standard deviation of three independent experiments $(\mathrm{n}=3) .{ }^{* *} \mathrm{P}<0.01$, compared with the negative control; ${ }^{*} \mathrm{P}<0.05$, compared with treatment with LPS alone. HUVECs, human umbilical vascular endothelial cells; CA, cynandione A; LPS, lipopolysaccharide. 
anti-adhesion effect of U937 monocytes to vascular endothelial cells by CA was the result of the inhibition of vascular inflammation, which prevented activation of the NF- $\kappa \mathrm{B}$ signaling pathway in HUVECs.

Chronic vascular inflammation is a main cause of various vascular inflammatory diseases. In vascular inflammatory sites, endothelial cells stimulated by inflammatory stimuli, including LPS, are known to interact with other leukocytes through a variety of adhesion molecules, including VCAM-1, inflammatory cytokines and chemokines $(2,26)$. The firm adhesion of leukocytes to the endothelium is critical in early atherosclerotic plaque development and in vascular inflammation. LPS is a component of the Gram-negative bacterial cell membrane and is known to induce inflammation in vivo. According to previous reports, LPS is known to increase the expression and secretion of cytokines, including IL-1 $\beta$, IL-6, IL-8, MCP-1 and TNF- $\alpha$, in HUVECs (27-29). IL-8 and MCP-1 are chemoattractant cytokines, which are involved in the accumulation of monocytes in vascular inflammation plaque lesions (30). TNF- $\alpha$ and other inflammatory stimuli have been reported to accrete the production of VCAM-1 in endothelial cell membranes, which can promote leukocyte-to-endothelium adhesion in vascular plaques (31). Therefore, inhibiting the secretion of pro-inflammatory cytokines and the expression of cell adhesion molecules may be important for the development of therapeutic agents for atherosclerosis caused by vascular inflammation.

The expression of VCAM-1, pro-inflammatory cytokines and chemoattractant cytokines, including IL-8 and MCP-1, in LPS-induced cells is regulated by the MAPK/IKK/IкB signaling cascade and activation of $N F-\kappa B(9,10,32)$. In addition, interactions with transcription factor binding elements, including NF- $\kappa \mathrm{B}$, can affect the activation of cell adhesion molecules and inflammatory mediators by LPS stimulation (32). In inflammatory conditions, the present study confirmed that CA inhibited the expression of VCAM-1 and pro-inflammatory cytokines. The results following signal inhibitor treatment demonstrated that ERK1/2 and p38 kinase were more important than JAK2 in the LPS-induced expression of VCAM-1. To characterize the mechanism underlying the effect of $\mathrm{CA}$ in LPS-induced HUVECs, the present study analyzed the phosphorylation pattern of the MAPK/NF- $\mathrm{BB}$ signaling pathway. CA markedly inhibited the phosphorylation of ERK $1 / 2$ and p38, and weakly inhibited the phosphorylation of JNK. This result indicated that $\mathrm{CA}$ served as a selective phosphorylation inhibitor of ERK1/2 and p38 kinase. It was also shown that CA affected the inhibition of the phosphorylation of IKK/I $\mathrm{B}-\alpha$, which is a downstream signaling pathway of MAPKs $(14,15)$. These data demonstrated that CA acted as a specific phosphorylation inhibitor of upstream molecules, including IKK and MAPKs, in the NF- $\mathrm{B}$ pathway.

In the resting state, the NF- $\kappa \mathrm{B}$ protein has two subunits, $\mathrm{p} 65$ and $\mathrm{p} 50$, which exist as a heterotrimer with $\mathrm{I} \kappa \mathrm{B}$ protein in the cytoplasm. In the presence of external stimuli, the I $\mathrm{B}$ protein is activated via phosphorylation, and the p65 subunit is released and translocated to the nucleus for target gene activation (33). The present study showed that CA inhibited nuclear translocation of the p65 subunit, and the results suggested that CA had an inhibitory effect on the transcriptional activity of $\mathrm{NF}-\kappa \mathrm{B}$ using a NF- $\kappa \mathrm{B} / \mathrm{Luc}$ reporter gene. The luciferase reporter assays demonstrated that CA suppressed the expression of the VCAM-1/Luc reporter gene in the LPS-stimulated HUVECs. The upregulation of VCAM-1 had functional effects, specifically the adhesion of monocytes to the endothelium in vascular inflammatory lesions. It was found that CA suppressed U937 monocyte adhesion to activated HUVECs by the inhibition of MAPK/IKK/I $\mathrm{B}-\alpha$, which correlated with the decreased expression of VCAM-1. These results provided additional evidence for the involvement of the MAPK/IKK/I $\mathrm{B} / \mathrm{NF}-\kappa \mathrm{B}$ signaling pathway in LPS-induced monocyte adhesion.

According to previous reports, natural compounds, including flavonoids, phytosteroids and small phenolic molecules, have various pharmacological activities, including anti-inflammatory activity $(34,35)$. CA is an acetophenone isolated from the roots of Cynanchum extracts, and is known to be a natural compound with effective anti-oxidant, anti-inflammatory and neuroprotective effects $(19,20)$.

In this present study, it was demonstrated that CA markedly inhibited the expression of LPS-induced VCAM-1 in HUVECs. The result showed that the LPS increased the expression levels of ICAM-1 and VCAM-1 in the HUVECs. However, it was confirmed that CA only inhibited the expression of VCAM-1 (Fig. 1). The activation of ICAM-1 by endotoxins, including LPS, in HUVECs has been demonstrated in a number of studies (36). In particular, the inhibition of cell adhesion molecules using $C$. wilfordii root ethanol extract and its active ingredients have shown no effect on the expression of ICAM-1 (18). Taken together, the results of the present and previous studies suggest that CA likely has no significant effect on the expression of ICAM-1. The present study further examined the inhibition of the expression of VCAM-1 at the molecular levels and suggested a mode of action in the anti-adhesion activity of CA. CA decreased the levels of IL-1 $\beta$, IL- 6 , TNF- $\alpha$, IL- 8 and MCP-1 induced by LPS. At the molecular level, $\mathrm{CA}$ acted to inhibit the activation of $\mathrm{NF}-\kappa \mathrm{B}$ by inhibiting the phosphorylation of IKK/IкB- $\alpha$, and inhibiting p38 and ERK1/2 MAPKs. In addition, CA substantially inhibited the adhesion of U937 to HUVECs in a dose-dependent manner. These results suggested that CA may be used as a novel drug candidate for the treatment of vascular inflammation by the inhibition of monocyte infiltration.

\section{Acknowledgements}

The present study was supported by funding from the Technology Commercialization Support Program (grant no. 814003031SB010), the Ministry of Agriculture Food and Rural Affairs (grant no. NRF-2015R1D1A1A01058506) and the Region Industry Total Information Service Program (grant no. R0004665), Republic of Korea.

\section{Competing interests}

The authors declare that they have no competing interests.

\section{References}

1. Binion DG, West GA, Ina K, Ziats NP, Emancipator SN and Fiocchi C: Enhanced leukocyte binding by intestinal microvascular endothelial cells in inflammatory bowel disease. Gastroenterology 112: 1895-1907, 1997. 
2. Calixto JB, Campos MM, Otuki MF and Santos AR Anti-inflammatory compounds of plant origin. Part II. modulation of pro-inflammatory cytokines, chemokines and adhesion molecules. Planta Med 70: 93-103, 2004.

3. Liu X, Pan L, Wang X, Gong Q and Zhu YZ: Leonurine protects against tumor necrosis factor- $\alpha$-mediated inflammation in human umbilical vein endothelial cells. Atherosclerosis 222: 34-42, 2012.

4. Luscinskas FW, Ding H, Tan P, Cumming D, Tedder TF and Gerritsen ME: L- and P-selectins, but not CD 49d (VLA-4) integrins, mediate monocyte initial attachment to TNF-alpha-activated vascular endothelium under flow in vitro. J Immunol 157 326-335, 1996.

5. Nizamutdinova IT, Kim YM, Jin H, Son KH, Lee JH, Chang KC and Kim HJ: Tanshinone IIA inhibits TNF- $\alpha$-mediated induction of VCAM-1 but not ICAM-1 through the regulation of GATA- 6 and IRF-1. Int Immunopharmacol 14: 650-657, 2012.

6. Alapati A, Deosarkar SP, Lanier OL, Qi C, Carlson GE, Burdick MM, Schwartz FL, McCall KD, Bergmeier SC and Goetz DJ: Simple modifications to methimazole that enhance its inhibitory effect on tumor necrosis factor- $\alpha$-induced vascular cell adhesion molecule-1 expression by human endothelial cells. Eur J Pharmacol 751: 59-66, 2015.

7. Qin P, Tang X, Elloso MM and Harnish DC: Bile acids induce adhesion molecule expression in endothelial cells through activation of reactive oxygen species, NF-kappaB, and p38. Am J Physiol Heart Circ Physiol 291: H741-H747, 2006.

8. Ueno H, Pradhan S, Schlessel D, Hirasawa H and Sumpio BE: Nicotine enhances human vascular endothelial cell expression of ICAM-1 and VCAM-1 via protein kinase C, p38 mitogen-activated protein kinase, NF-kappaB, and AP-1. Cardiovasc Toxicol 6 : $39-50,2006$

9. Chai H, Wang Q, Huang L, Xie T and Fu Y: Ginsenoside Rb1 inhibits tumor necrosis factor-alpha-induced vascular cell adhesion molecule-1 expression in human endothelial cells. Biol Pharm Bull 31: 2050-2056, 2008.

10. Zapolska-Downar D and Naruszewicz M: Propionate reduces the cytokine-induced VCAM-1 and ICAM-1 expression by inhibiting nuclear factor-kappa B (NF-kappaB) activation. J Physiol Pharmacol 60: 123-131, 2009.

11. Wang W, Deng M, Liu X, Ai W, Tang Q and Hu J: TLR4 activation induces nontolerant inflammatory response in endothelial cells. Inflammation 34: 509-518, 2011.

12. Li B, Zhang R, Li J, Zhang L, Ding G, Luo P, He S, Dong Y, Jiang W, Lu Y, et al: Antimalarial artesunate protects sepsis model mice against heat-killed Escherichia coli challenge by decreasing TLR4, TLR9 mRNA expressions and transcription factor NF-kappa B activation. Int Immunopharmacol 8: 379-389, 2008.

13. Mo SJ, Son EW, Lee SR, Lee SM, Shin DH and Pyo S: CML-1 inhibits TNF-alpha-induced NF-kappaB activation and adhesion molecule expression in endothelial cells through inhibition of IкBalpha kinase. J Ethnopharmacol 109: 78-86, 2007.

14. Madonna R, Massaro M,Pandolfi A,Consoli A and De Caterina R The prominent role of $\mathrm{p} 38$ mitogen-activated protein kinase in insulin-mediated enhancement of VCAM-1 expression in endothelial cells. Int J Immunopathol Pharmacol 20: 539-555, 2007.

15. Suna S, Sakata Y, Shimizu M, Nakatani D, Usami M, Matsumoto S, Mizuno H, Ozaki K, Takashima S, Takeda H, et al Lymphotoxin-alpha3 mediates monocyte-endothelial interaction by TNFR I/NF-kappaB signaling. Biochem Biophys Res Commun 379: 374-378, 2009.

16. Kim MS, Baek JH, Park JA, Hwang BY, Kim SE, Lee JJ and Kim KW: Wilfoside K1N isolated from Cynanchum wilfordi inhibits angiogenesis and tumor cell invasion. Int $\mathrm{J}$ Oncol 26 : $1533-1539,2005$

17. Choi DH, Lee YJ, Kim JS, Kang DG and Lee HS: Cynanchum wilfordii ameliorates hypertension and endothelial dysfunction in rats fed with high fat/cholesterol diets. Immunopharmacol Immunotoxicol 34: 4-11, 2012.

18. Koo HJ, Sohn EH, Pyo S, Woo HG, Park DW, Ham YM, Jang SA, Park SY and Kang SC: An ethanol root extract of Cynanchum wilfordii containing acetophenones suppresses the expression of VCAM-1 and ICAM-1 in TNF- $\alpha$-stimulated human aortic smooth muscle cells through the NF- $\mathrm{B}$ pathway. Int $\mathrm{J}$ Mol Med 35: 915-924, 2015.

19. Hwang BY, Kim YH, Ro JS, Lee KS and Lee JJ: Acetophenones from the roots of Cynanchum wilfordii H(EMSLEY). Arch Pharm Res 22: 72-74, 1999.
20. Jiang Y, Choi HG, Li Y, Park YM, Lee JH, Kim DH, Lee JH, Son JK, Na M and Lee SH: Chemical constituents of Cynanchum wilfordii and the chemotaxonomy of two species of the family Asclepiadacease, $C$. wilfordii and $C$. auriculatum. Arch Pharm Res 34: 2021-2027, 2011.

21. Lee MK, Yeo H, Kim J and Kim YC: Protection of rat hepatocytes exposed to $\mathrm{CCl} 4$ in-vitro by cynandione $\mathrm{A}$, a biacetophenone from Cynanchum wilfordii. J Pharm Pharmacol 52: 341-345, 2000.

22. Yang SB, Lee SM, Park JH, Lee TH, Baek NI, Park HJ, Lee H and Kim J: Cynandione A from Cynanchum wilfordii attenuates the production of inflammatory mediators in LPS-induced BV-2 microglial cells via NF- $\mathrm{KB}$ inactivation. Biol Pharm Bull 37: 1390-1396, 2014

23. Kim SH, Lee TH, Lee SM, Park JH, Park KH, Jung M, Jung H, Mohamed MA, Baek NI, Chung IS and Kim J: Cynandione A attenuates lipopolysaccharide-induced production of inflammatory mediators via MAPK inhibition and NF- $\kappa \mathrm{B}$ inactivation in RAW264.7 macrophages and protects mice against endotoxin shock. Exp Biol Med 240: 946-954, 2015.

24. Park KH, Lee TH, Kim CW and Kim J: Enhancement of CCL15 expression and monocyte adhesion to endothelial cells (ECs) after hypoxia/reoxygenation and induction of ICAM-1 expression by CCL15 via the JAK2/STAT3 pathway in ECs. J Immunol 190: 6550-6558, 2013

25. Chen CC, Rosenbloom CL, Anderson DC and Manning AM: Selective inhibition of E-selectin, vascular cell adhesion molecule-1, and intercellular adhesion molecule-1 expression by inhibitors of I kappa B-alpha phosphorylation. J Immunol 155: 3538-3545, 1995

26. Choi JH, Yoo JY, Kim SO, Yoo SE and Oh GT: KR-31543 reduces the production of proinflammatory molecules in human endothelial cells and monocytes and attenuates atherosclerosis in mouse model. Exp Mol Med 44: 733-739, 2012.

27. Weglarz L, Dzierzewicz Z, Skop B, Orchel A, Parfiniewicz B, Wiśniowska B, Swiatkowska L and Wilczok T: Desulfovibrio desulfuricans lipopolysaccharides induce endothelial cell IL-6 and IL-8 secretion and E-selectin and VCAM-1 expression. Cell Mol Biol Lett 8: 991-1003, 2003.

28. Makó V, Czúcz J, Weiszhár Z, Herczenik E, Matkó J, Prohászka Z and Cervenak L: Proinflammatory activation pattern of human umbilical vein endothelial cells induced by IL-1 $\beta$, TNF- $\alpha$, and LPS. Cytometry A 77: 962-970, 2010.

29. Fernández-Pisonero I, Dueñas AI, Barreiro O, Montero O, Sánchez-Madrid F and García-Rodríguez C: Lipopolysaccharide and sphingosine-1-phosphate cooperate to induce inflammatory molecules and leukocyte adhesion in endothelial cells. J Immunol 189: 5402-5410, 2012.

30. Lukacs NW, Strieter RM, Elner V, Evanoff HL, Burdick MD and Kunkel SL: Production of chemokines, interleukin-8 and monocyte chemoattractant protein-1, during monocyte: Endothelial cell interactions. Blood 86: 2767-2773, 1995.

31. Madonna R, Massaro M and De Caterina R: Insulin potentiates cytokine-induced VCAM-1 expression in human endothelial cells. Biochim Biophys Acta 1782: 511-516, 2008.

32. Ming X, Ding M, Zhai B, Xiao L, Piao T and Liu M: Biochanin A inhibits lipopolysaccharide-induced inflammation in human umbilical vein endothelial cells. Life Sci 136: 36-41, 2015.

33. Lush CW, Cepinskas G and Kvietys PR: LPS tolerance in human endothelial cells: Reduced PMN adhesion, E-selectin expression, and NF-kappaB mobilization. Am J Physiol Heart Circ Physiol 278: H853-H861, 2000.

34. Jung MR, Lee TH, Bang MH, Kim H, Son Y, Chung DK and Kim J: Suppression of thymus- and activation-regulated chemokine (TARC/CCL17) production by $3-O-\beta$-D-glucopyanosylsp inasterol via blocking NF- $\mathrm{BB}$ and STAT1 signaling pathways in TNF- $\alpha$ and IFN- $\gamma$-induced HaCaT keratinocytes. Biochem Biophys Res Commun 427: 236-241, 2012.

35. Lee TH, Jung M, Bang MH, Chung DK and Kim J: Inhibitory effects of a spinasterol glycoside on lipopolysaccharide-induced production of nitric oxide and proinflammatory cytokines via down-regulating MAP kinase pathways and NF- $\mathrm{KB}$ activation in RAW264.7 macrophage cells. Int Immunopharmacol 13: 264-270, 2012

36. Lee CH, Reid YA, Jong JS and Kang YH: Lipopolysaccharide-induced differential cell surface expression of intercellular adhesion molecule-1 in cultured human umbilical cord vein endothelial cells. Shock 3: 96-101, 1995. 